\title{
Tunable microfluidic microlenses
}

\author{
Armin Werber and Hans Zappe
}

\begin{abstract}
A novel type of liquid microlens, bounded by a microfabricated, distensible membrane and activated by a microfluidic liquid-handling system, is presented. By use of an elastomer membrane fabricated by spin coating onto a dry-etched silicon substrate, the liquid-filled cavity acts as a lens whereby applied pressure changes the membrane distension and thus the focal length. Both plano-convex and plano-concave lenses, individual elements as well as arrays, were fabricated and tested. The lens surface roughness was seen to be $\sim 9 \mathrm{~nm} \mathrm{rms}$, and the focal length could be tuned from 1 to $18 \mathrm{~mm}$. This lens represents a robust, self-contained tunable optical structure suitable for use in, for example, a medical environment. (C) 2005 Optical Society of America

OCIS codes: $350.3950,220.3620,220.3630,010.1080$.
\end{abstract}

\section{Introduction}

Microlenses with intrinsic tunability are of interest for a wide variety of applications, particularly beam shaping, optical interconnection, and imaging. ${ }^{1}$ The last-named application is of particular relevance for endoscopy, in which a variable imaging system provides an advantage for tissue imaging; no dynamic micro-optical systems suitable for use in extremely small fiber endoscopes, with diameters of as little as $0.5 \mathrm{~mm}$ at the distal end, have been demonstrated. Further application areas include biochip readers, ${ }^{2}$ which require microlens arrays that are completely tunable, and beam shaping for optical switching. ${ }^{3}$

A limited number of concepts for tunable microlenses have been demonstrated by diverse approaches and technologies, such as by electro-wetting,, 45 through the use of liquid-crystal microlenses, ${ }^{6}$ casting of poly(dimethyl siloxane) (PDMS), ${ }^{7,8}$ and adaptive lenses driven by radiation pressure. ${ }^{9}$ The limitations on liquid lenses tuned by the electrowetting approach include requirement for high driving voltages and the requirement that only polar liquids be used. The liquid-crystal technique is hampered by the possibility of nonuniformities in the electric field, which could lead to optical aberrations. Moreover,

The authors are with Laboratory for Micro-optics, Institute of Microsystem Technology, University of Freiburg, Georges-KöhlerAllee 102, 79110 Freiburg, Germany. A. Werber's e-mail address is werber@imtek.de.

Received 15 October 2004; revised manuscript received 20 December 2004; accepted 20 December 2004.

0003-6935/05/163238-08\$15.00/0

(C) 2005 Optical Society of America both methods require the presence of electrodes, which can disturb the optical performance. The casting method, finally, requires more than one substrate, leading to alignment and adhesion problems; the PDMS cast of the master substrate has to be aligned and fixed to the lens's body such that misalignment and poor reproducibility frequently result. And the radiation-pressure-driven liquid-liquid interface lenses require a high-power laser for generation of the lens meniscus.

In the present research, a novel type of microfluidic microlens is presented that overcomes a number of these limitations. As shown in Fig. 1, the membranebased, liquid-filled tunable microlenses employ a $50-\mu \mathrm{m}$-thick, pressure-actuated PDMS membrane structured upon a silicon fluidic chip. This chip is mounted onto a Pyrex substrate, thereby forming a fluidic cavity filled with a liquid of suitable refractive index. The body of the lens is then formed by the liquid, whereby the membrane curvature provides the lens shape. Varying the pressure of the liquid by using the fluidic system changes the shape of the membrane and thus the optical power of the lens. As shown in the figure, both plano-convex and planoconcave lenses may be obtained. A microphotograph of two distended, $400-\mu \mathrm{m}$-diameter convex lenses is shown in Fig. 2.

\section{Design and Fabrication}

The membrane-based microlens is fabricated on a silicon substrate bonded to a Pyrex substrate; the membrane itself is a spin-coated PDMS layer on the front side of the silicon wafer. The use of these materials means that no cavity deformation takes place when pressure is applied and no critical alignment or 


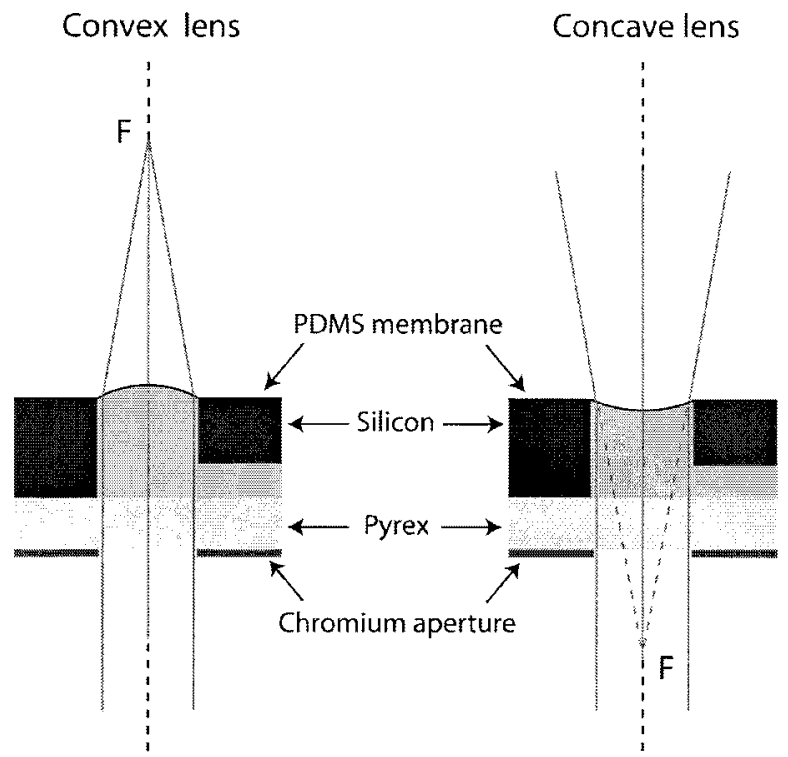

Fig. 1. Cross-sectional diagram of a membrane-based microfluidic microlens, showing its implementation as plano-convex and plano-concave lenses. The drawing is to scale for a microlens with diameter $d=400 \mu \mathrm{m}$.

adhesion problems occur. The use of standard microelectromechanical systems processing techniques, under clean-room conditions to ensure particle-free optical surfaces, implies that low-cost mass fabrication of these lenses is possible.

Microlens fabrication is thus based on silicon process technology combined with a number of nonsilicon materials to yield the complete structure; a summary of the process is shown schematically in Fig. 3. Processing begins with photolithography on a silicon wafer polished on both sides in which the lens openings and the microfluidic channels are defined. In wet oxidation and subsequent plasma-enhanced chemical-vapor deposition oxidation steps, a masking layer is deposited. This layer is structured by photolithography and subsequently opened by reactive ion etching (RIE). The first oxide mask defines the con-

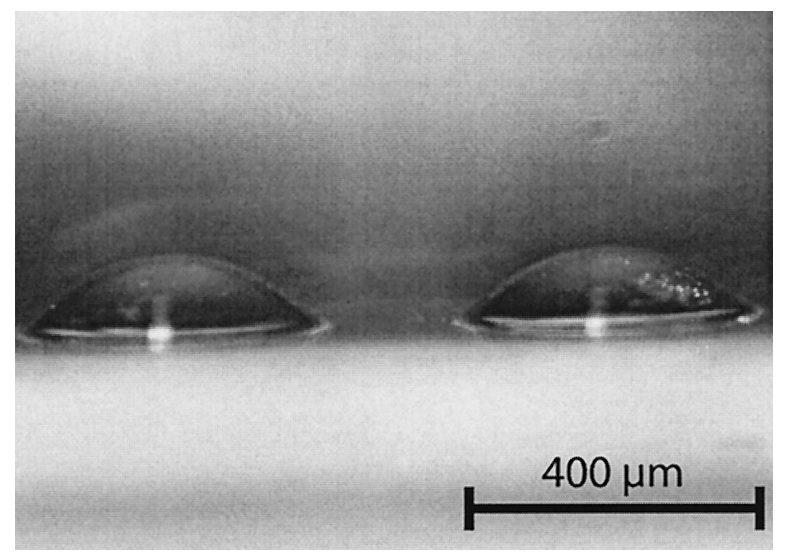

Fig. 2. Microphotograph of two distended plano-convex lenses achieved by application of positive fluidic pressure. The lens diameter of $400 \mu \mathrm{m}$ is shown.
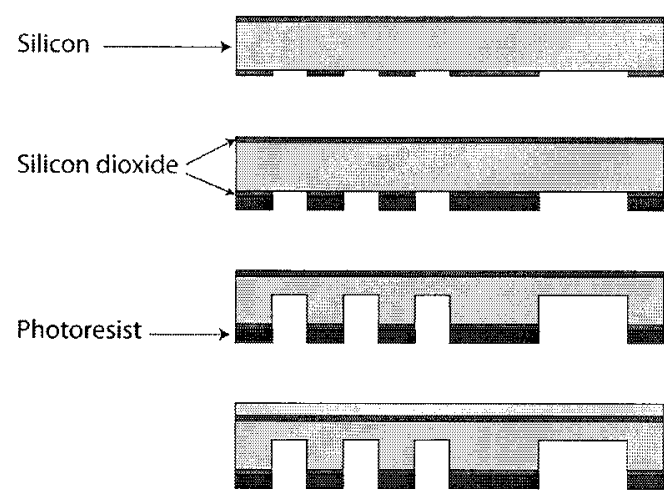

PDMS
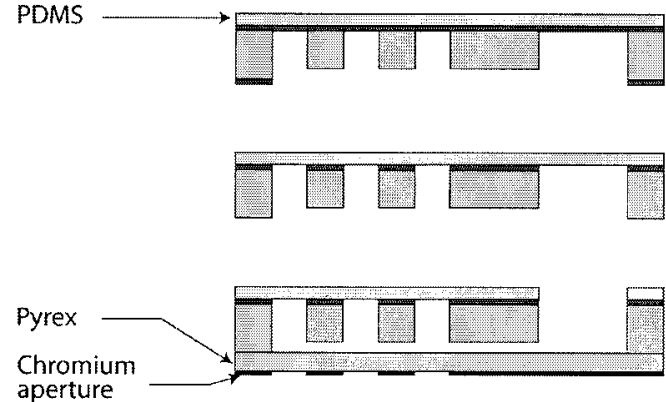

(g) aperture

Liquid

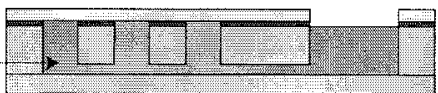

(h)

Fig. 3. Process summary: (a) Silicon wafer with both side $\mathrm{SiO}_{2}$ layer and with back side opened by RIE; (b) photoresist, spin coated and structured; (c) first ICP RIE etching; (d) spin coating of primer and PDMS; (e) second ICP RIE etching; (f) $\mathrm{SiO}_{2}$ removal; (g) bonding of the Pyrex wafer and patterning of the back-side chromium layer; (h) sawing and filling of the devices.

tours of the lens chamber, the fluidic channels, and the reservoir, with a typical structure as shown in Fig. 4. Circular lens openings with diameters of 300-600 $\mu \mathrm{m}$ were defined along with the fluidic channels and the reservoirs. In a second photolithography step, a $12-\mu \mathrm{m}$-thick photoresist layer is patterned. This second mask step defines the contours of the lens chambers and the reservoirs.

Defined by the second photoresist mask, the lens chambers and the reservoirs are then etched through half of the silicon wafer by an inductively-coupled

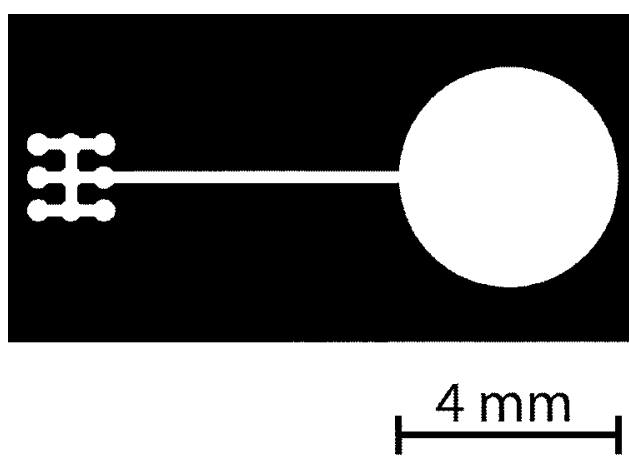

Fig. 4. Layout for a lens array with $400-\mu \mathrm{m}$ lenses. 
plasma (ICP) RIE process. Afterward, the resist layer is removed in a plasma asher. This step is followed by front-side spin coating of a thin PDMS film. We used Sylgard 186 PDMS material from Dow Corning. To improve the adhesion of the PDMS to the silicon or silicon oxide, the substrate is coated with a primer, DC 92-023 from Dow Corning, of $\sim 3-\mu \mathrm{m}$ thickness. The thickness of the PDMS film is $\sim 50 \mu \mathrm{m}$, measured with a surface profiler. This film is cured for 15 min at $150{ }^{\circ} \mathrm{C}$. This PDMS or silicone elastomer film forms the pressure-actuated membrane, which encloses the working liquid and ultimately defines the lens's curvature.

As the lenses are arranged as arrays with different dimensions and contours, one fluidic reservoir will provide nine lenses with the required working liquid and pressure; in the future each lens will be controlled by an independent microfluidic system. In a second ICP RIE etch step the lens chambers, the reservoirs, and the fluidic channels are etched simultaneously, passivated by the structured $\mathrm{SiO}_{2}$ layer. The etching stops abruptly when the $\mathrm{SiO}_{2}$ layer at the front side is reached because the etch selectivity ${ }^{10}$ to $\mathrm{SiO}_{2}$ is approximately 200 to 1 .

Finally, the front-side $\mathrm{SiO}_{2}$ layer is removed in a last RIE step, resulting in the PDMS membrane's now being freely stretched over the silicon lens chamber. To enclose the liquid cavity, a thin Pyrex wafer is subsequently bonded to the back side of the silicon substrate to cap the fluidic channels and chambers. Bonding is accomplished by a UV-curable optical adhesive, spun on as a very thin layer of $\sim 3 \mu \mathrm{m}$ onto the Pyrex wafer. After alignment, a UV flood exposure followed by an anneal at a temperature of $50^{\circ} \mathrm{C}$ for $24 \mathrm{~h}$ results in covalent bonds between the Pyrex and the silicon substrates, and this ensures that the structure remains mechanically stable.

Because the Pyrex wafer is fully transparent, not only the lens chambers but also the fluidic channels and the reservoirs are visible from the back of the bonded structure. To minimize scattering and cross talk between the single lenses of the array, an aperture stop on the unpatterned Pyrex wafer is required. Such an aperture is made by use of a 100-nm-thick vapor-deposited chrome layer, subsequently structured by a wet etch.

The wafer stack is then separated into individual chips, each with an array of nine lenses and one reservoir, by sawing. During this last step, the delicate PDMS membrane must be protected by a photoresist layer to prevent scratching during chip separation. The protective resist coating is stripped in acetone, and the chips rinsed in deionized water.

To prepare the fluidic lenses for operation, one has to introduce the liquid optical medium into the microfluidic chambers; one accomplishes this filling by taking advantage of capillary forces that essentially result in a self-filling of the cavities. Two liquid media were employed: a mixture of $50-$ wt. $\%$ water and 50-wt. \% ethanol and a proprietary liquid from Cargille with a high refractive index $(n=1.600)$. In the former case the addition of ethanol is important to reduce surface tension and thus provide better filling, caused by capillary forces. The $\mathrm{H}_{2} \mathrm{O}$ :ethanol mixture working liquid provides a refractive index of $n=1.3534$, measured with a refractometer. Cavity filling is accomplished in a vacuum chamber to deaerate the liquid enclosed inside the small fluidic channels. Any remaining bubbles quickly diffuse out of the system because the PDMS membrane is gas permeable.

The devices are operated by use of a silicone tube glued to the fluidic reservoir entrance with UVcurable adhesive. A manually operated syringe and a calibrated pressure sensor were then used to define the pressure exerted on the liquid, thereby leading to the desired lens distension.

\section{Characterization of the Microlenses}

Characterization of the membrane microlenses was accomplished by use of a number of tools. Surface profile measurements were made with a white-light interferometer (Zygolot New View 5022) or a mechanical surface profiler (Tencor P-11). The focal length of the microlenses was measured directly with a Zeiss Axioplan2 optical microscope and Axiovision software to change the focal plane dynamically.

Using the white-light interferometer, we determined the surface roughness of the tunable lenses to be $\sim 9 \mathrm{~nm}$ (rms). This is an excellent value, particularly when one considers that this is a micromechanical structure with a large surface-to-volume ratio. We used the same measurement setup to measure the lens's radius of curvature, from which the focal length could be calculated, as a function of the applied pressure. The pressure of the liquid optical medium was measured with a Motorola MPX5050 pressure sensor with a resolution of $90 \mathrm{mV} / \mathrm{kPa}$. Determination of the maximum lens height allowed the radius of curvature to be calculated through

$$
R=\frac{d^{2}}{8 h}+\frac{h}{2}
$$

where $R$ is the radius of the lens, $d$ is the lens diameter, and $h$ is the maximum height. The focal length could then be determined simply by

$$
f=\frac{R}{\left(n_{1}-1\right)}
$$

where $f$ is the focal length and $n_{1}$ is the refractive index of the liquid optical medium. These expressions are based on the thin-lens approximation and a spherical lens profile.

The pressure range in the measurement series varied from 0 to $54 \mathrm{kPa}$, giving the results shown in Fig. 5 . For the highest pressures, the curvature radius saturates at $\sim 0.2 \mathrm{~mm}$, with controllable values ranging from 0.2 to $6 \mathrm{~mm}$. We discuss its effect on focal length below.

For comparison, the lens curvature was also mea- 


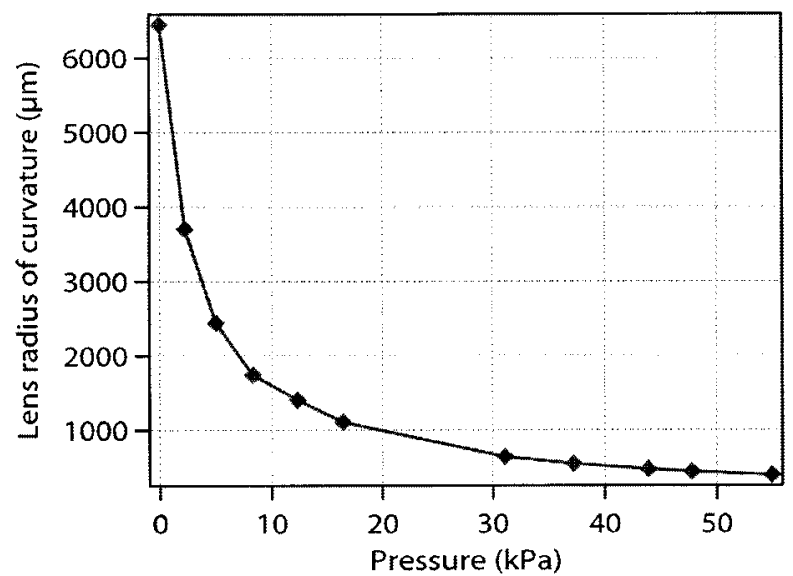

Fig. 5. Lens radius of curvature as a function of pressure applied to the liquid optical medium (ethanol and water) as measured by white-light interferometry.

sured by use of a mechanical surface profiler. In this case a needle with a tip radius of $2 \mu \mathrm{m}$ and a cone angle of $60^{\circ}$ mechanically scans the surface; because the PDMS film is soft, the scanning force was minimized and had a value of $\sim 1 \mathrm{mN}$. The measurement tip must be aligned such that the full diameter of the lens is scanned, thereby giving a true measure of the lens's shape.

As can be seen from Fig. 6, the lens profile was measured as a function of applied pressure in the range 4-54 kPa; the corresponding radii of curvature are given in Fig. 5. The lens profile is not ideally spherical for two reasons: the nonlinear deflection of the PDMS membrane and the fixed edge of the membrane, which leads to an inflection point at the lens edge. In the latter case the lens curvature at positive pressure is concave instead of convex, as would be the case for a completely spherical lens. But an additional aperture can minimize the aberrations that are due to the lens edge. The nonlinearity of the elastic PDMS membrane, however, is an intrinsic effect of the material and is not alterable.

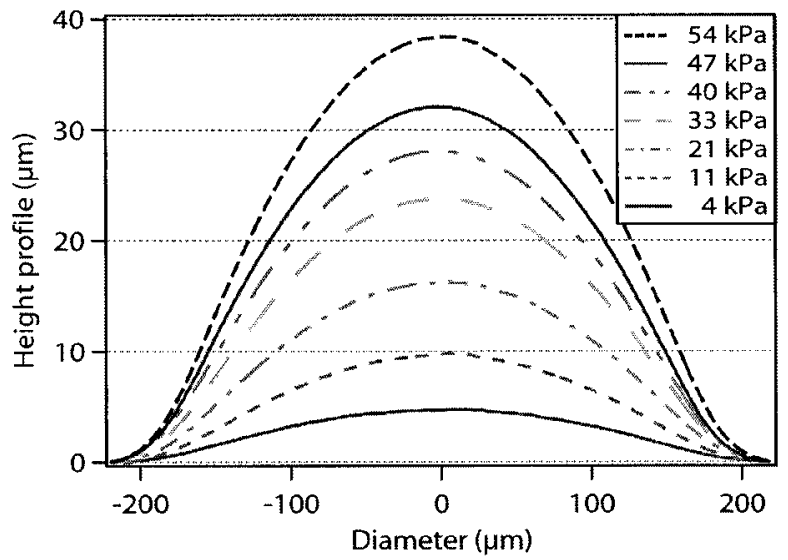

Fig. 6. Complete lens profile measured by a mechanical profiler for a 500- $\mu \mathrm{m}$-diameter lens as a function of applied pressure in the range $4-54 \mathrm{kPa}$.

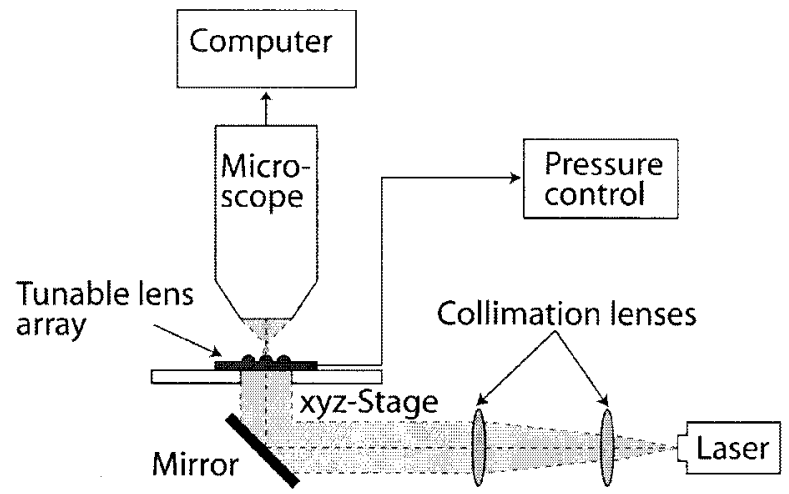

Fig. 7. Measurement setup for focal-length measurement. A collimated laser beam illuminates the lens array from the back side. The light is focused by each lens, such that the lenses of the array are tested in transmission. One determines the focal length for an individual lens by moving the translation stage in the $z$ direction at that point where the beam waist is minimal. The amount by which the translation stage was moved corresponds to the focal length.

Focal length was measured directly with the experimental setup shown in Fig. 7. In this measurement the lens array is illuminated with a collimated, expanded laser beam (at $\lambda=550 \mathrm{~nm}$ ) from the back (i.e., Pyrex) side of the structure, corresponding to the planar side of the plano-convex lens. The collimated light is then focused by each lens of the two-dimensional array; the lenses are thus tested in transmission, corresponding to the normal mode of operation. One then determines the focal length for an individual lens by moving the automated translation stage from focus on the wafer surface to the position where minimum beam waist is achieved; the total translation then corresponds to the focal length.

The focal length is shown in Fig. 8 as a function of pressure measured with this approach; for comparison, the focal length as determined from application of Eqs. (1) and (2) to the surface profile data of Fig. 6 is also shown. The measurements were performed with the two liquid optical media available, with $n_{1}$

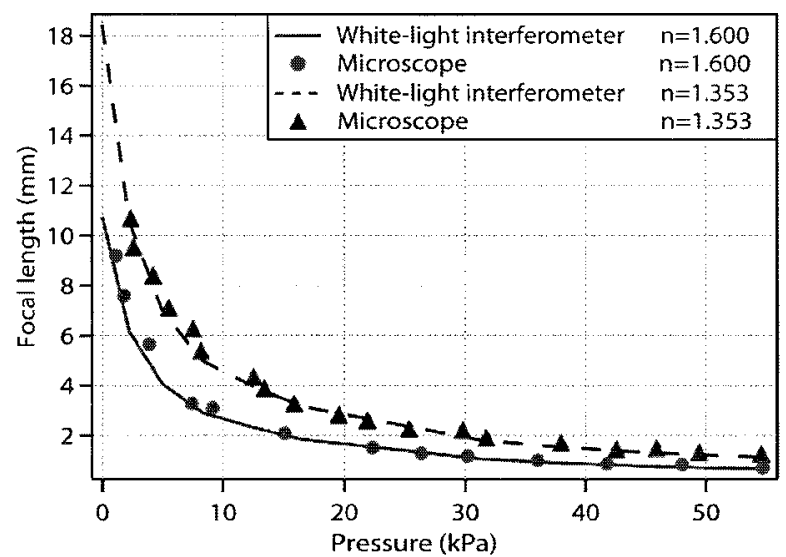

Fig. 8. Focal length of the membrane lenses as a function of applied pressure as measured directly and as calculated from optical surface profile measurements. 


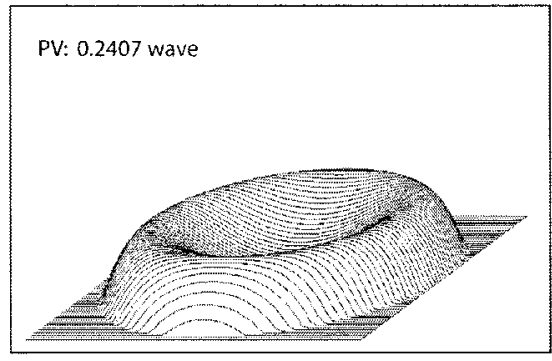

Wave-front function

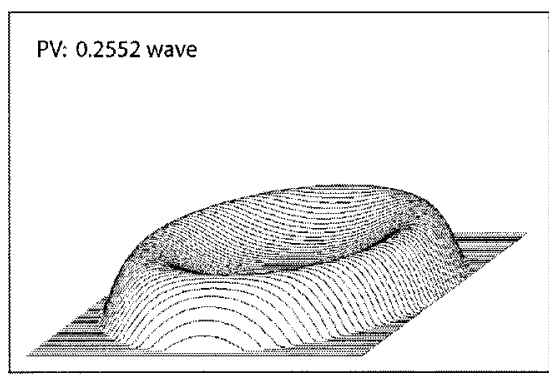

Wave-front function

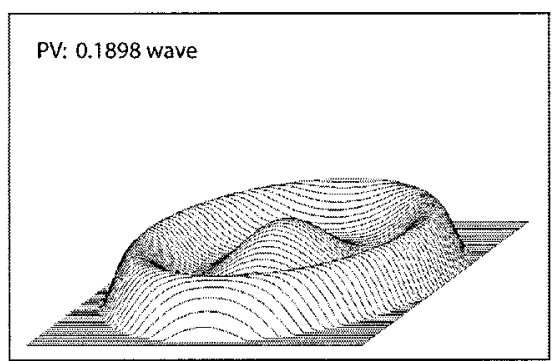

Wave-front function

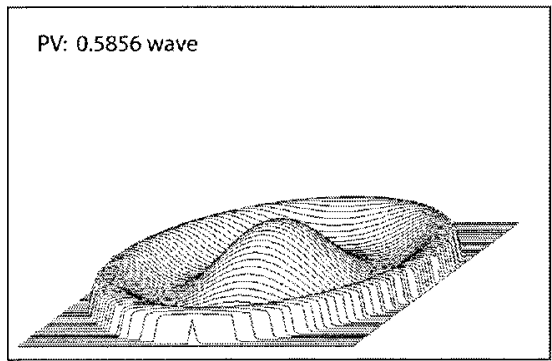

Wave-front function

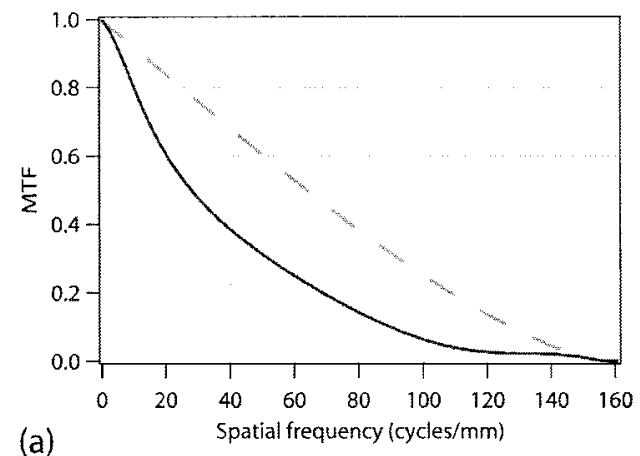

(a)
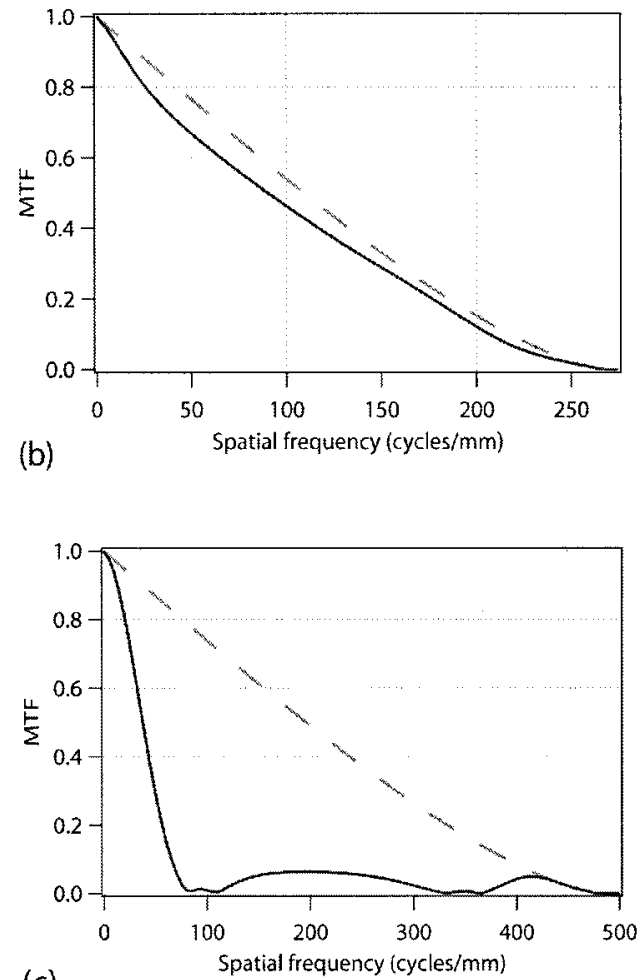

(c)

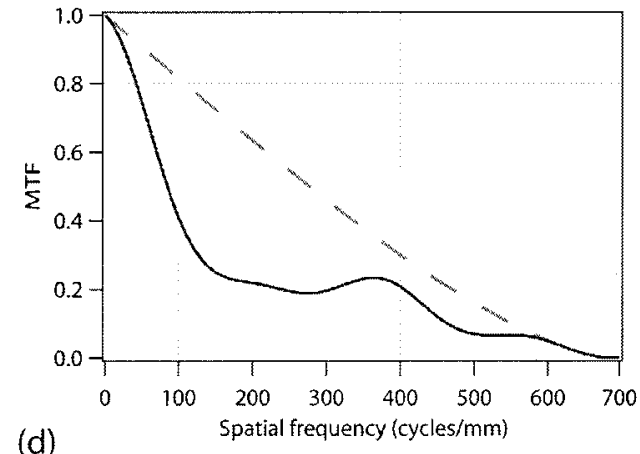

(d)

Fig. 9. Wave fronts and MTFs at several pressures: (a) 11, (b) 21, (c) 40, and (d) $54 \mathrm{kPa}$. The wavelength in this simulation was determined at $\lambda=550 \mathrm{~nm}$. Dashed curves show the diffraction limits. PV, peak to valley.

$=1.35$ and $n_{1}=1.6$. It can be seen that the focal length varies from 1 to $18 \mathrm{~mm}$, saturating at $\sim 1 \mathrm{~mm}$ for high pressures. The two characterization approaches yield values in close agreement, supporting our assertion that the lens profiles are close to spherical.

The lens aberration was evaluated by use of the topological data acquired by the surface profiler. 
Table 1. Spherical Aberrations

\begin{tabular}{cccc}
\hline & \multicolumn{3}{c}{ Aberration $^{a}$} \\
\cline { 2 - 4 } Pressure $(\mathrm{kPa})$ & First Order $(\lambda)$ & Second Order $(\lambda)$ & Third Order $(\lambda)$ \\
\hline 11 & -0.144 & -0.021 & 0.004 \\
21 & -0.137 & -0.048 & 0.013 \\
40 & -0.039 & -0.106 & 0.029 \\
54 & 0.217 & -0.225 & 0.014 \\
\hline
\end{tabular}

${ }^{a}$ Spherical aberrations of first, second, and third orders as functions of four pressure values. The aberrations are rms values, given in waves for a wavelength of $550 \mathrm{~nm}$.

These data were approximated by a sixth-order polynomial describing the membrane surface at several pressure values. Subsequently a model of a tunable membrane microlens was implemented with the Zemax optical design program. The model comprised three parts: a $200-\mu \mathrm{m}$-thick Pyrex layer, a liquid lens chamber, and the deflected membrane. The membrane surface was represented as an even asphere by use of the polynomial coefficients derived above. The refractive index of the Pyrex layer was $n_{P}=1.4731$, and that of for the liquid was $n_{1}=1.6057$, both at a wavelength of $546.1 \mathrm{~nm}$. To minimize the aberration caused by the lens edge we used a $300-\mu \mathrm{m}$ aperture in the simulation.

The simulation was made at four pressure values: $11,21,40$, and $54 \mathrm{kPa}$. Figure 9 shows the wave front and the modulation transfer function (MTF) for these pressures. Based on the aberrations, the Zemax program calculates a list of the Zernike coefficients by using Zernike fringe polynomials. The coefficients represent the spherical aberrations of first, second, and third orders. Table 1 illustrates the spherical aberrations based on the Zernike coefficient data, and Table 2 shows the peak-to-valley aberration of the different wave fronts, based on Fig. 9.

\section{Discussion}

A decided advantage of this tunable lens design compared with competing tunable microlens concepts is the ability to vary lens curvature through a range of positive as well as negative values. Application of positive pressure results in plano-convex lenses, and by application of negative pressure the lenses become plano-concave. These two operating modes are illustrated in Fig. 10 for a membrane with $300-\mu \mathrm{m}$ diameter operated at 4 and $0.2 \mathrm{kPa}$. A limited distension

Table 2. Wave-Front Aberrations

\begin{tabular}{cc}
\hline & $\begin{array}{c}\text { Wave Front } \\
\text { Aberration } \\
(\lambda)^{a}\end{array}$ \\
\hline 11 & 0.2407 \\
21 & 0.2552 \\
40 & 0.1898 \\
54 & 0.5856 \\
\hline
\end{tabular}

${ }^{a}$ Peak-to-valley aberrations of the wave fronts of Fig. 9. The aberrations are given in waves for a wavelength of $550 \mathrm{~nm}$.
$(3.5 \mu \mathrm{m})$ was used for this measurement because the white-light interferometer is unable to measure profiles steeper than $\sim 7.73^{\circ}$ from the horizontal.

Further optimization of the lens structure, particularly with respect to membrane thickness and elasticity, as well as the range and accuracy of pressures required for achievement of a desired lens shape, requires a more-detailed understanding of the statics of the membrane. To this end, was employed the Ansys finite-element modeling package to simulate the membrane deflection as a function of various parameters; a typical result, for a membrane with an elasticity modulus of $4 \mathrm{MPa}$, a thickness of $50 \mu \mathrm{m}$, and a diameter of $400 \mu \mathrm{m}$, is shown in Fig. 11. A comparison of lens distension as measured by data is shown in Fig. 12. The consistently lower magnitudes of distension as measured by the surface profiler are due to the fact that this approach distorts the mem-

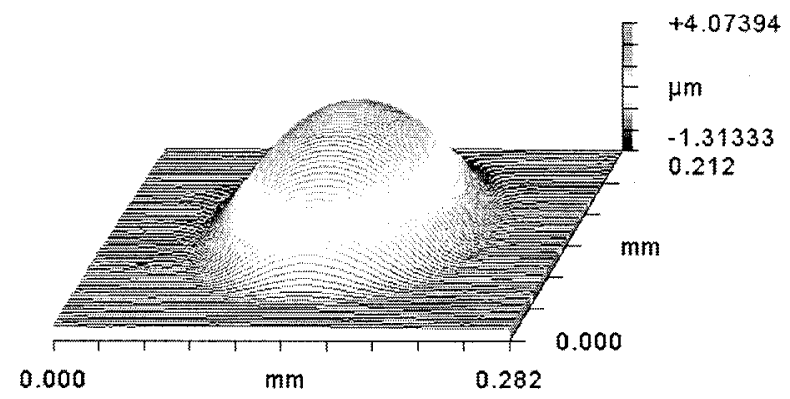

(a)

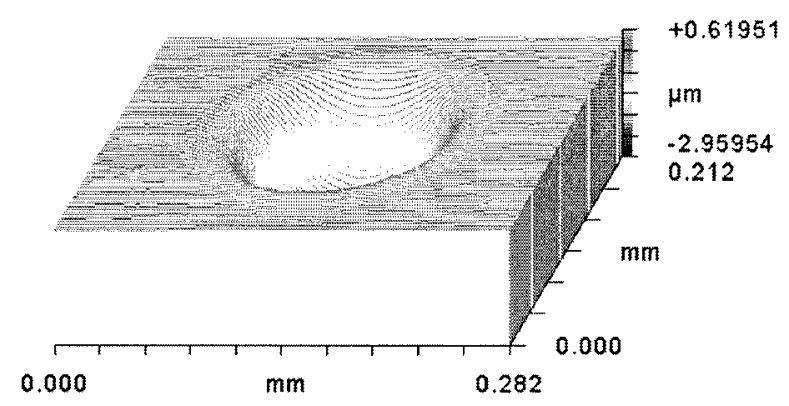

(b)

Fig. 10. Contours measured with a white-light interferometer for the tunable lenses operated in (a) plano-convex and (b) planoconcave modes; the measurements are for a $300-\mu \mathrm{m}$ diameter lens operated at 4 and $0.2 \mathrm{kPa}$, respectively. 

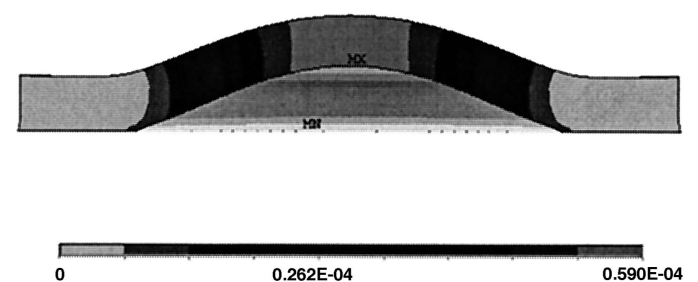

Fig. 11. Typical Ansys result for a distended membrane, $50 \mu \mathrm{m}$ thick, with a pressure of $50 \mathrm{kPa}$.

brane, despite the low pressure of the stylus. As a result, the measured results are smaller than the simulated and interferometry-based values.

Simulation results for lens height profile as a function of pressure are shown in Fig. 13. The simulated height profiles are similar to the measured data shown in Fig. 6. The gradient of curvature at the lens edge agrees with the experimentally acquired curvatures. The lens height varies moderately between the

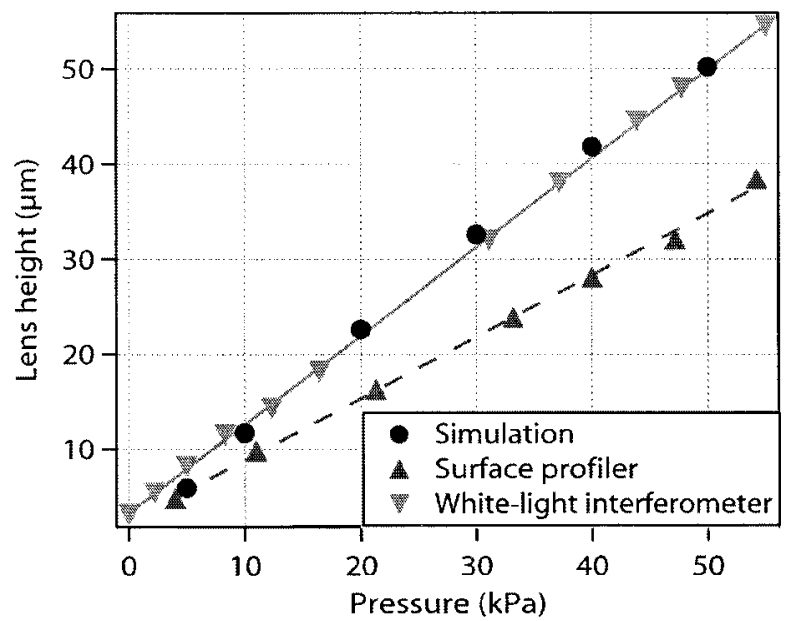

Fig. 12. Comparison of maximum lens distension as a function of pressure as measured by interferometry and surface profiling and compared with simulation results.

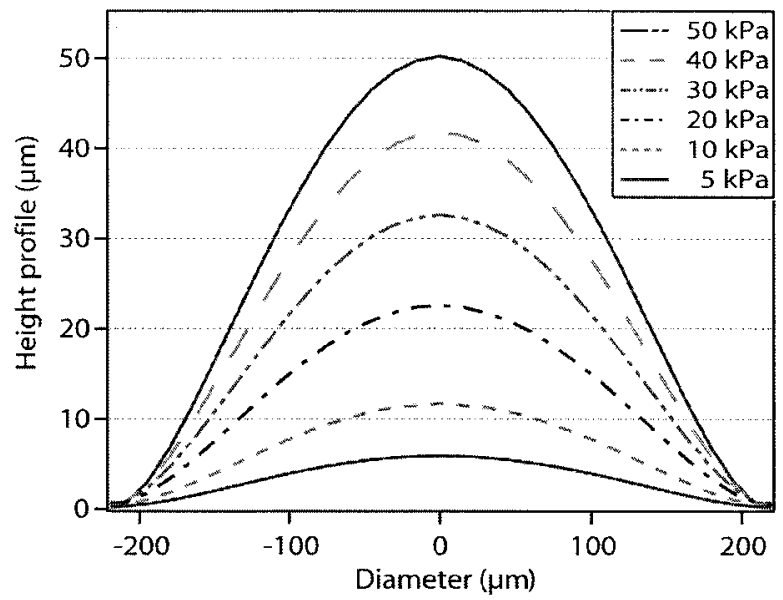

Fig. 13. Lens profile as predicted by an Ansys simulation for a $50-\mu \mathrm{m}$-thick PDMS membrane with $400-\mu \mathrm{m}$ diameter. simulation and the experiment because of distortion caused by the surface profiler.

The minimization of spherical aberration is a key aspect of membrane lens design. The maximum firstorder spherical aberration was obtained at a pressure of $54 \mathrm{kPa}$. This value of $0.217 \lambda$ at a wavelength of $550 \mathrm{~nm}$ is acceptable. But this value can be achieved only with a predefined aperture of $300 \mu \mathrm{m}$, applied to a lens with a diameter of $400 \mu \mathrm{m}$. This aperture blanks the nonspherical areas at the lens edges and thus improves the optical performance. As can be seen from Table 1 and Fig. 9, the spherical aberration decreases with decreasing pressure.

Simulation of the MTF, as illustrated in Fig. 9, confirms these aberration results. Two curves are plotted for each pressure value: the dashed curves in Fig. 9 depict the diffraction limit and depict the best MTF value for the lens aperture, limited only by diffraction; the solid curves below are the real MTFs, determined for the membrane surface. The best contrast is achieved with the lens at a pressure value of $20 \mathrm{kPa}$, as shown in Fig. 9(b). The worst contrast is found for the pressure of $54 \mathrm{kPa}$, as shown in Fig. 9(d). The resolution, represented as the maximum spatial frequency, depends on the numerical aperture of the lenses, so the resolution increases with higher numerical aperture and thus increases with increasing pressure.

Further design considerations are the means for application of pressure, pressure variations, repeatability, and aging. The application of constant pressure over an extended period of time can be achieved only by use of a pressure controller with a precise microvalve. Maintaining constant pressure is important to prevent variations in the focal length during the imaging period. The repeatability and aging effects have not yet been investigated in detail, but we have not observed any hysteresis in membrane deflection, especially with low maximum pressure values. Extremely high pressures may overstretch the PDMS membrane, causing plastic deformation and thus leading to nonreproducibility effects.

Finally, the PDMS membrane is stable and flexible in a temperature range from $-50^{\circ} \mathrm{C}$ to $+200{ }^{\circ} \mathrm{C}$, but the stability of the refractive-index liquid must also be considered. Volatile liquid combinations, such as $\mathrm{H}_{2} \mathrm{O}$ :ethanol mixtures, are therefore not suitable for long-term use in such a lens system.

\section{Summary}

A new liquid microlens, bounded by a microfabricated, distensible membrane and activated by a microfluidic liquid-handling system, has been described and characterized. The fully enclosed structure and pressure-dependent mode of operation allow the lens to be tuned over a wide range of positive and negative curvatures, such that a micro-optical element with tunability over a wide range results. As the liquid optical medium of the lens is fully enclosed and needs to be transparent only at the operating wavelength, a wide range of materials, with a correspondingly wide range of refractive indices as well as dispersive 
power, may be employed in future generations of the device.

The lens technology also permits the assembly of multicomponent lenses, such as tunable achromatic doublets. Through the use of appropriate liquid optical media, tunable achromatic microlens systems may be fabricated, providing even greater flexibility for a variety of applications.

\section{References}

1. D. Daly, Microlens Arrays (Taylor \& Francis, New York, 2001).

2. J. C. Roulet, R. Völkel, H. P. Herzig, E. Verpoorte, N. F. de Rooij, and R. Dändliker, "Microlens systems for fluorescence detection in chemical microsystems," Opt. Eng. 40, 814-821 (2001).

3. H.-P. Herzig, Micro-optics (Taylor \& Francis, New York, 1997).

4. B. Berge and J. Perseux, "Variable focal lens controlled by an external voltage: an application of electrowetting," Eur. Phys. 3, 159-163 (2000).

5. T. Krupenkin, S. Yang, and P. Mach, "Tunable liquid microlens," Appl. Phys. Lett. 82, 316-318 (2003).

6. H. Ren, Y.-H. Fan, and S.-T. Wu, "Liquid-crystal microlens arrays using patterned polymer networks," Opt. Lett. 29, 1608-1610 (2004).

7. J. Chen, W. Wang, J. Fang, and K. Varahramyan, "Variablefocusing microlens with microfluidic chip," Micromech. Microeng. 14, 675-680 (2004).

8. N. Chronis, G. L. Liu, K.-H. Jeong, and L. P. Lee, "Tunable liquid-filled microlens array integrated with microfluidic network," Opt. Express 19, 2370-2378 (2003), http://www. opticsexpress.org.

9. A. Casner and J.-P. Delville, "Adaptive lensing driven by the radiation pressure of a continuous-wave laser wave upon a near-critical liquid-liquid interface," Opt. Lett. 26, 1418-1420 (2001).

10. M. Madou, Fundamentals of Microfabrication (CRC Press, Boca Raton, Fla., 2002). 